\title{
Local Shape Modelling Using Warplets
}

\author{
Abhir Bhalerao and Roland Wilson \\ Department of Computer Science, University of Warwick, UK \\ \{abhir, rgw\}@dcs.warwick.ac.uk
}

\begin{abstract}
We develop a statistical shape model for the analysis of local shape variation. In particular, we consider models of shapes that exhibit self-similarity along their contours such as fractal and space filling curves. Overlapping contour segments are parametrically modelled using an orthogonal basis set, Legendre Polynomials, and used to estimate similarity transformations to a reference segment, which may or may not be from the contour being analysed. The alignment is affine and regresses the model to the data by least squares fitting and is followed by a PCA of the coregistered set of contour segments. The local shape space is defined jointly by the segment-to-segment 'warps' and the mean plus eigen vectors of the shape space, hence Warplets. The parametric modelling makes the alignment correspondence-free so that arbitrary sized segments can be aligned and the local warps can be inverted to reconstruct model approximations of the data. The approach shows potential in capturing fine details of shape variation and is applicable to complex shapes and those with repetitive structure, when only a few training examples are available.
\end{abstract}

\section{Introduction}

Statistical shape models are built from a set of training examples and, by Principal Component Analysis (PCA), the eigen modes of the model represent the most likely variations of the shape [1]. Such models have been used in object segmentation to constrain the variation of a deformable template [2]. Two important issues normally arise with such models: the size of the training set and the need for point-to-point correspondences in the training samples (homology). The former problem exacerbates the latter as it is tedious to hand label homologous points for larger training samples and complex shapes need greater numbers of points. Much work has focussed on these problems in recent years, e.g. [3,4]. However, for certain classes of shapes where there are periodic variations, e.g folding patterns of cortical geometry, these global or lumped parameter models ([5]) are inadequate or become impractical to use. Many 'active' shape model (ASM) based segmentation methods therefore defer to the physical constraints for the final refinement steps when registering a deformable template to such a class of shapes. Hybrid shape models have been proposed that attempt to incorporate models of local shape variation into the global scheme. For example, multiresolution analysis by wavelet 
transforms combined with fractal priors [5] or with a PCA [4], or curve evolution based on scale-spaces [6]. These models strive to separate the fine detail of the shape variation from the large scale changes to leverage either stability for the physical deformations or greater power in the lower modes of variation.

Here we describe a locally parameterised statistical shape model, the contour Warplet. By explicitly modelling each segment with Legendre polynomials, the affine pose estimation can be performed by regression. The warplets are subsequently decomposed by PCA in the normal way and by retaining the pose parameters, we can reconstruct the entire contour to measure the segment-tosegment variation.

\section{Method}

\subsection{Localising the Contour}

A contour, $C$, can be described as a set of $N$ points in the plane, $\mathbf{c}_{i}=\left(x_{i}, y_{i}\right)^{T}, 0 \leq$ $i<N-1$. Without loss of generality, we consider point sets with sizes in powers of two; $N=2^{e}$, e.g, $N=512$. The contour can be divided into equal sections of size, $B=2^{f}$, such that $2 \leq f<e$, made to overlap by half their lengths $B / 2$. For example, a contour of size 512 can be divided into 32 overlapping segments of size $B=32$. Let segment $j$ be denoted by $S_{j}=\left\{\mathbf{c}_{i}\right\}, j B / 2 \leq i \leq$ $(j+1) B / 2$. If the final segment is made to straddle the $B / 2$ points at the end and the first $B / 2$ points in the start of $C$, the contour is regarded as being closed.

To reconstruct the contour, the segment coordinates are windowed by ${\operatorname{a~} \cos ^{2}()}^{2}$ function centred on the middle point and the windowed coordinates of the overlapping sections, $S W_{j}$, are simply summed in to the output. For $n_{s}$ segments, the reconstructed contour is calculated by

$$
C=\sum_{j=0}^{n_{s}-1}\left\{\cos ^{2}\left((i-B / 2) \frac{\pi}{2}\right) \mathbf{c}_{i}\right\}_{j} .
$$

The reason this works is that the window function shifts by $\frac{\pi}{2}$ for each half segment, hence $\cos ^{2}()$ becomes $\sin ^{2}()$ and in the overlap region points proportionally sum to 1 , Figure 1.

\subsection{Contour Modelling}

Each contour segment, $S_{j}$, is parameterised in polar form transforming points $\mathbf{c}_{i}$ to $\mathbf{p}_{i}=\left(\rho_{i}, \theta_{i}\right)^{T}$, where $\rho_{i}=\sqrt{\left(x_{i}-x_{o}\right)^{2}+\left(y_{i}-y_{o}\right)^{2}}$ and $\theta_{i}=\tan ^{-1}\left(\left(y_{i}-\right.\right.$ $\left.\left.y_{o}\right) /\left(x_{i}-x_{o}\right)\right)$. If the contour is centred on the origin, i.e. $\sum_{i} \mathbf{c}_{i}=\mathbf{0}$, then $\left(x_{o}, y_{o}\right)^{T}=\mathbf{0}$ and the polar parameters represent the radial and angular variations of the curve around the origin. Polar coordinates are convenient for the subsequent modelling as the contours of interest will be more or less circular. 

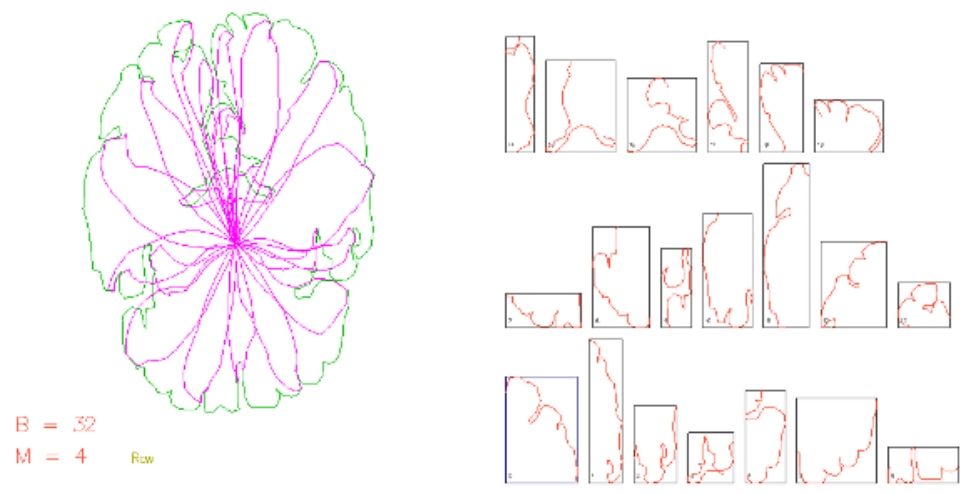

Fig. 1. Localisation of contour into segments of size $B=32$. The original contour is shown in green on the left-hand images together with the windowed segments which form loops centred on the origin in magenta. The segmented sections are shown in the right-hand window

The Legendre Polynomials (LP) derive from solving Legendre's differential equation and are defined for the range, $x \in[-1,1]$ as follows:

$$
P_{n}(x)=\frac{1}{2^{n} n !} \frac{d^{n}}{d x^{n}}\left(x^{2}-1\right)^{n}
$$

where $n$ is the polynomial order.

$$
P_{0}(x)=1, \quad P_{1}(x)=1, \quad P_{2}(x)=\frac{1}{2}\left(3 x^{2}-1\right), \quad P_{3}(x)=\frac{1}{2}\left(5 x^{3}-3 x\right) .
$$

These function form an orthogonal basis set in the interval $-1 \leq x \leq 1$ since

$$
\int_{-1}^{1} P_{m}(x) P_{n}(x) d x=0, m \neq n .
$$

Note that these polynomials can be efficiently generated by the recurrence formula:

$$
P_{n+1}(x)=\frac{(2 n+1) x P_{n}(x)-n P_{n-1}(x)}{(n+1)},
$$

and fitting a function, say $f(x)$, by $(n+1)$ coefficients can be accomplished by projection onto the basis set. We model all contour segments, $S_{j}$, by fitting polynomials of a given order $M$, to the radial and angle functions of the polar parameterisation functions of each segment. For example, the radial function is approximated by the weighted sum as follows:

$$
\hat{\rho}(x)=\sum_{m=0}^{M-1} l_{m} P_{m}(x), \quad \rho(x)=\rho_{i}, \quad x=\left(i-\frac{B}{2}\right) /\left(\frac{B}{2}\right)
$$



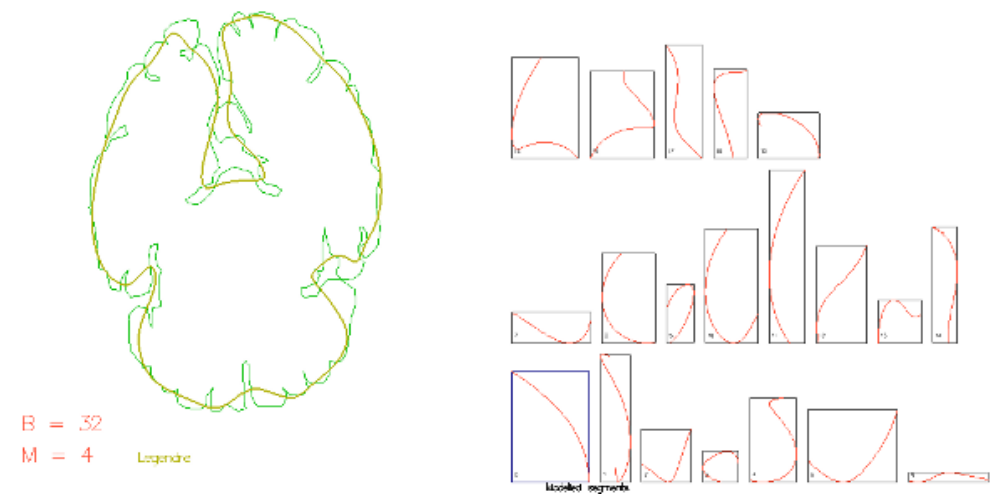

Fig. 2. Examples of Legendre Polynomial models of contour segments for $M=4$. The yellow contour is the model reconstruction. The right-hand images show the individual model segments for $B=32$

where $l_{m}$ result from the linear regression. The angle function $\hat{\theta}(x)$ is similarly modelled. Figure 2 illustrates the LP contour modelling for the two previous example contours for $M=4$. The right-hand images show the estimated model for segments of size $B=32$ and the yellow contour on the left-hand image is the model reconstruction using overlapping weighted segments. In these examples, each contour segment is coded by $2(M+1)$ coefficients plus the centre of arc coordinates $(\mathbf{0})$. In figure 3 model reconstruction for a range or segment sizes for $M=3$ are shown with errors given in RMS point distances under each figure. Lengthening the segments for a given order smoothens the shape representation in quite a natural way and is reminiscent of curve-evolution approaches using scale-spaces. The LP representation is fundamentally free of the number of points in each segment. For all the examples presented here, we set $B$ to be the same size for each segment.

$B=32$

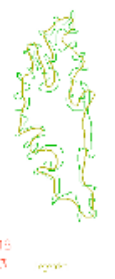

11.1

$$
B=64
$$

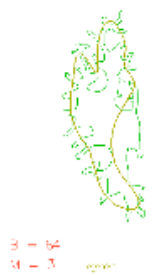

16.7

$$
B=128
$$$$
B=256
$$

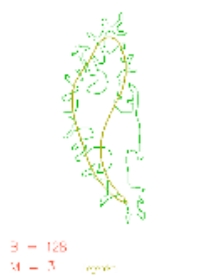

26.5

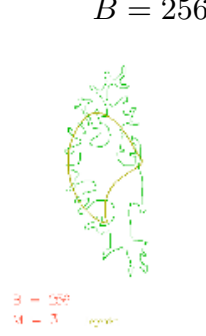

43.9

Fig. 3. Effects of segment length $B$ for a given LP model order $(M=3)$. Examples show results on a convex white matter contour of size approximately $256 \times$ 512. RMS contour position errors are given below each figure 


\subsection{Procrustes Registration}

The analysis of shape variation across the contour segments requires them to be registered to a common coordinate frame: the Kendall Shape Space [7]. By registration, the segments are corrected for position, rotation and scale variation. Here, an affine warp is estimated by transforming the modelled segments $\hat{S}_{j}$ to some prototype segment $S_{k}$. Dryden [7] notes that affine matching using regression reduces to linear regression for any number of dimensions. Thus we can satisfactorily separate the $2 \mathrm{D}$ pose alignment problem into two, $1 \mathrm{D}$ regressions for the polar coordinate functions $\theta_{j}(x), \rho_{j}(x)$ of each segment, $S_{j}$. Let the warped segment be $W_{j}$, then define warps of the form:

$$
W_{j}=T_{A}\left(\hat{S}_{j}\right)=\left(\begin{array}{cc}
a_{2 \rho}+a_{1 \rho} x & 0 \\
0 & a_{2 \theta}+a_{1 \theta} x
\end{array}\right) \hat{\mathbf{p}}_{i}+\left(\begin{array}{c}
a_{0 \rho} \\
a_{0 \theta}
\end{array}\right)
$$

The warps, $S_{j} \rightarrow S_{k}$, from all segments to a chosen prototype segment, $S_{k}$ are sought. These are each represented by 6 transformation parameters, $A_{j k}=$ $\left(\mathbf{a}_{\rho}, \mathbf{a}_{\theta}\right)$. For example, the radial model function for segment $j$ is transformed by

$$
W_{j \rho}=\left(a_{2 \rho}+a_{1 \rho} x\right) \hat{\rho}(x)+a_{0 \rho}
$$

As with the LP model fitting, this yields a system of equations for each model point in $\hat{S}_{j}$ against a target data point in the prototype segment, $S_{k}$ and the system can be solved by SVD. It is perhaps useful to briefly comment on the effects of some simple similarity transformations on a typical segment. For a rotation of a segment, we require an estimate only of an angular displacement, $a_{0 \theta} \neq 0$. A constant radial scaling will be represented by $a_{1 \rho}$ with $a_{2 \rho}=0$, while a linear scaling across the segment will be accounted for by a non-zero term: $\left(a_{2 \rho}+a_{1 \rho} x\right)$.

To unwarp a segment back to the contour space, we trivially apply the inverse mapping,

$$
T_{\rho}^{-1}\left(W_{j \rho}\right)=\frac{\left(W_{j \rho}-a_{0 \rho}\right)}{\left(a_{2 \rho}+a_{1 \rho} x\right)},
$$

and similarly for the warped segment angle function using $T_{\theta}^{-1}$.

\subsection{Warplets and Affine Shape Spaces}

Having registered all contour segments to a reference segment, $S_{k}$, a PCA is used in the standard way to encode the shape variation. Denoting the decomposition as

$$
\hat{W}_{j}=M_{k}+\sum_{d=1}^{D} \Gamma_{j d} . \Phi_{k d},
$$

where $M_{k}$ is the mean warplet and $\Gamma_{j}$ are the weighting for each of the $D$ eigen vectors, $\Phi_{k}$, associated with non-zero eigen values. The contour warplet is defined jointly by the regression parameters of the Procrustes alignment, $A_{j k}$, and the coordinates of the segment in the tangent shape space:

$$
\mathcal{W}_{j k}=\left\{A_{j k}, \boldsymbol{\Gamma}_{j k}\right\}
$$


If the segments are evenly distributed around the mean, then the tangent space distribution is even also and should be independent of the registration. However, a full Procrustes analysis requires a pair-wise registration between shapes, $S_{j}, S_{k}$ for all $j, k$. Thus, the choice of the prototype shape, $S_{k}$ for $\mathcal{W}$ is debatable, but iterative optimisation is possible (e.g. as in [8]).

\section{$3 \quad$ Experiments}

Figure 4 shows three examples of warplet shape spaces for the synthetic 'wavy' curve, a grey-matter brain contour hand-drawn from a slice of an MR brain data set, and a hand-drawn white-matter contour. All contours were analysed using 32 point segments starting at an arbitrary position and modelled with 4 Legendre Polynomials per segment $M=4$, i.e. a cubic fit. The blue-box on the left-hand images shows the bounds of the prototype segment onto which all others were warped; warped segments are shown together with their extents on the right-hand images (these images have been rescaled). Each of these warped segments become input shapes to a PCA analysis. In the top two examples, the scaling and rotation of the segments can be seen to roughly match the prototype. On the white-matter contour, the alignment results are harder to interpret. After PCA, we can plot the sizes of the principal non-zero eigen values and, in figure 5, we have plotted these for PCA without alignment and with alignment to the segment that produced the most 'compact' shape space for the white-matter example, i.e. the plot which gave the least variation from the mean.

In figure 6 , the first few principal modes, $d<D$, have been plotted for the grey-matter contour (middle row figure 4) and shown in proportions of $\sqrt{\lambda_{d}}$ around each mean, $M_{k}$. The mean is in the first box on each row and then the modes in order across the page. As expected, most of the variability is in the first 4 or 5 modes around 2 standard-deviations around the mean. Some of the finer contour variation is apparent in the higher modes, whereas the lower modes show largely elongation and shrinking not accounted for by the warping. Finally, in figure 7 we reconstruct the contour using equation (9) after taking increasing numbers of modes up to $D$. Next to each result is a RMS point error between the shape model approximation (shown in black) and the original contour (shown in green). Where the segments deviate in shape from the prototype (shown in the blue box on each image), we see that the contour reconstruction curve lies far from the true contour. It is only beyond mode $d=4$ that the reconstruction errors fall below about 10 pixels.

\section{Conclusions}

We have described a new statistical shape model for local contour modelling and analysis, contour warplets, which has some unique properties. Our experiments thus far have been limited to a small set of arbitrarily chosen curves, some 

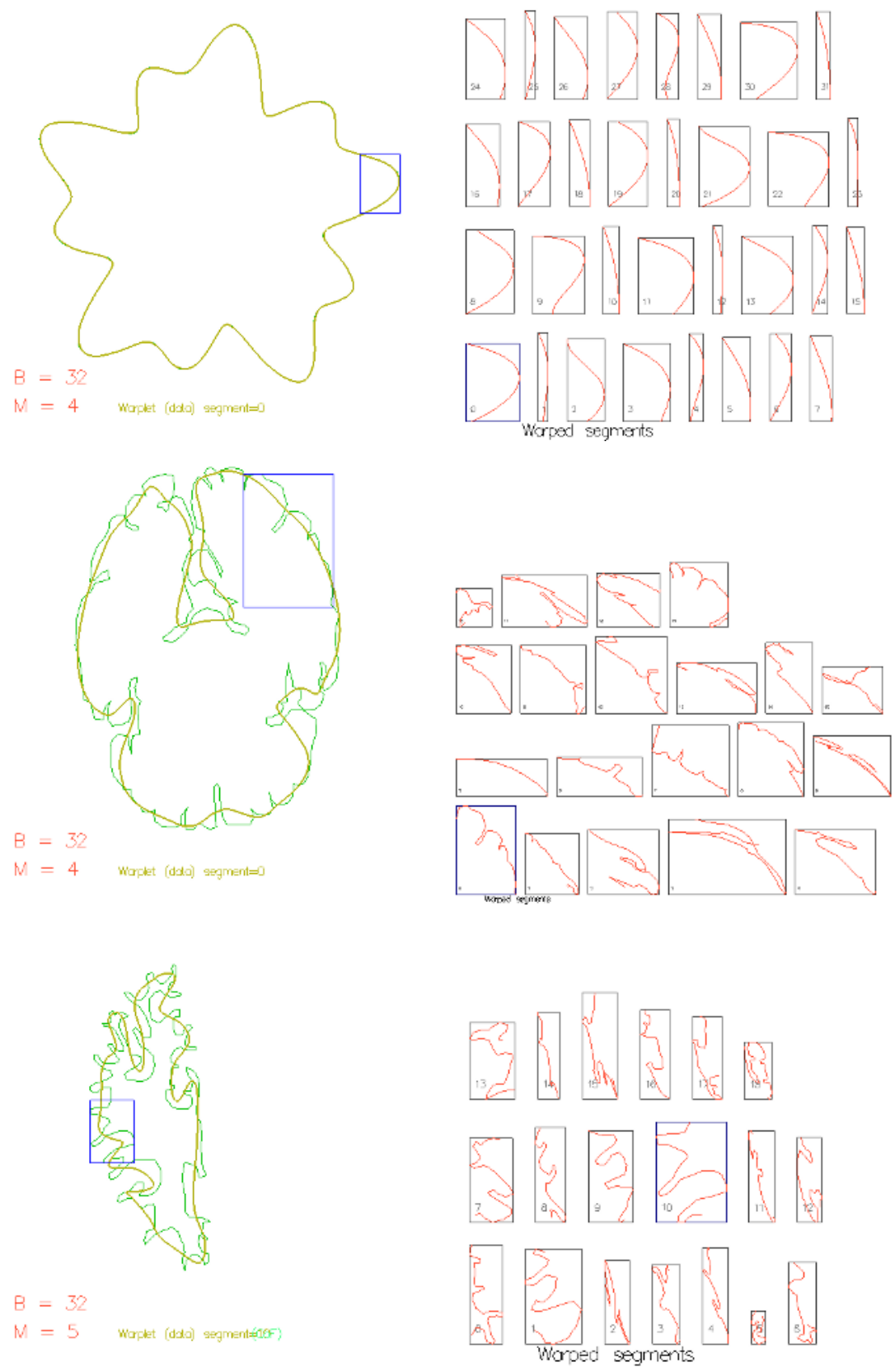

Fig. 4. Contour segments after alignment. The rectangles marked in blue on the left-hand image shows the bounding-box of the prototype segment against which all are registered. Registered segments shown in right-hand images (the order of presentation is left-to-right, bottom-to-top). White-matter contours (bottom two rows) have been parameterised from estimated centres-of-arc (see 2.1) 


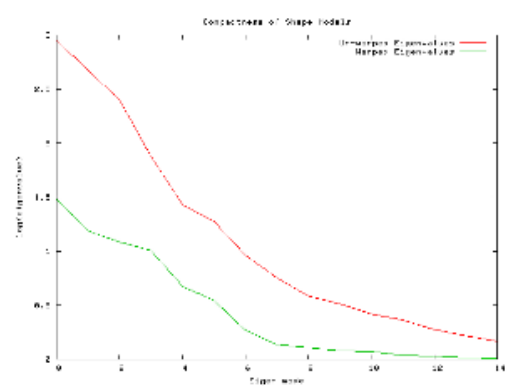

Fig. 5. Comparison of warplet PCA model against unregistered model. Log plot of eigenvalues against number of principal mode. The PCA on unregistered segments (red) gives an upper-bound for this curve (warplet PCA in green)

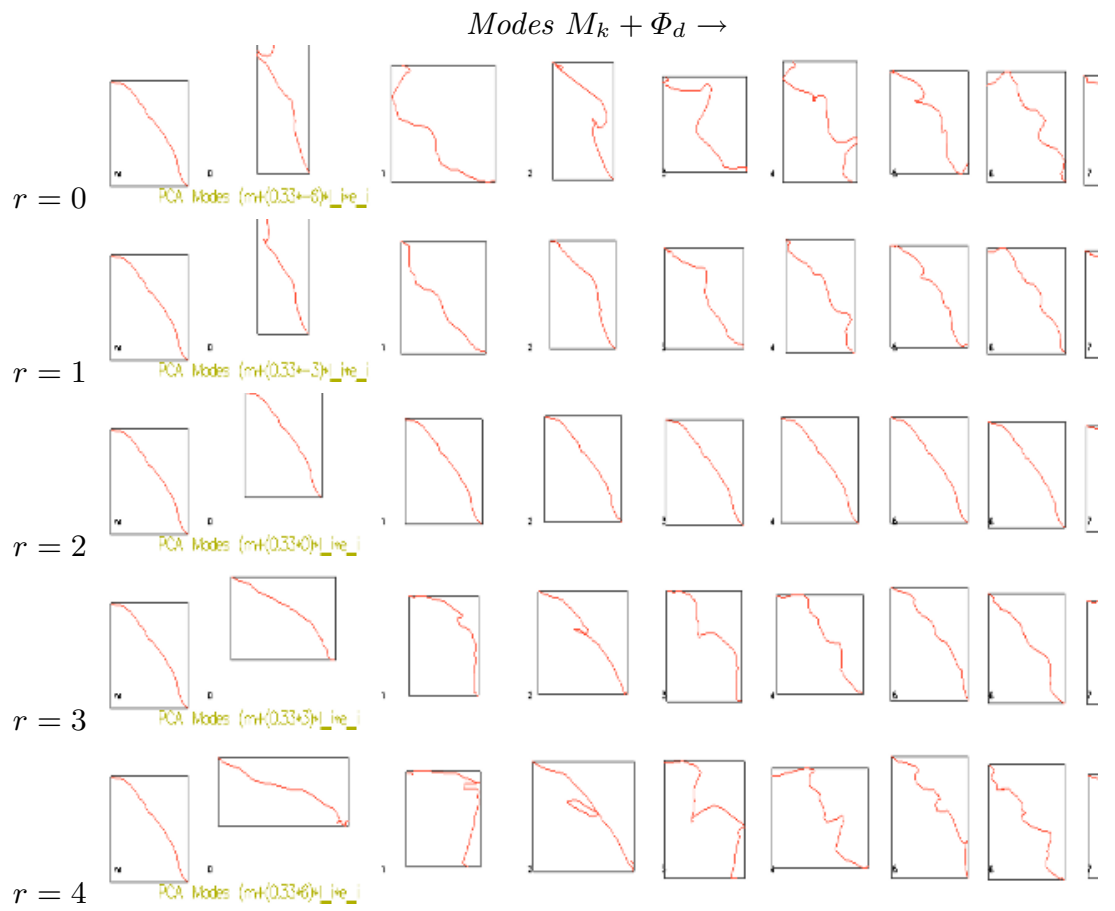

Fig. 6. Mean and eigen modes of aligned segments. Shown for entire brain contour (shown in middle row, figure 4). Each row, $0 \leq r \leq 4$, shows the principal non-zero modes, $d$, as $M_{k}+(r-2) \sqrt{\lambda_{k d}} \Phi_{k d}$

of which exhibit the characteristics we claim to be able to succinctly capture. We suggest that the model could be usefully applied to study brain morphology $[9,10]$. For example, Mangin et al. [11] note the fundamental problem of establishing sulcal-based homologies between subject brains so that the gyri can be compared. Perhaps, a warplet description of the folding could provide a 


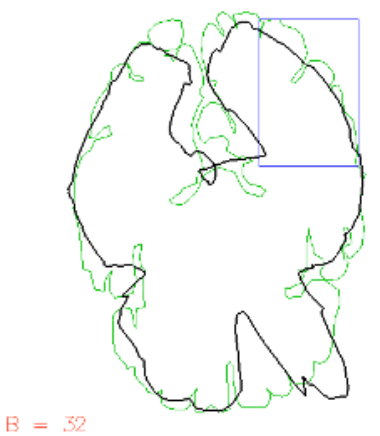

32.3

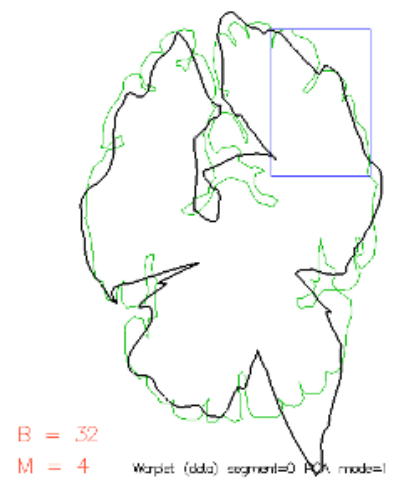

23.7

19.5
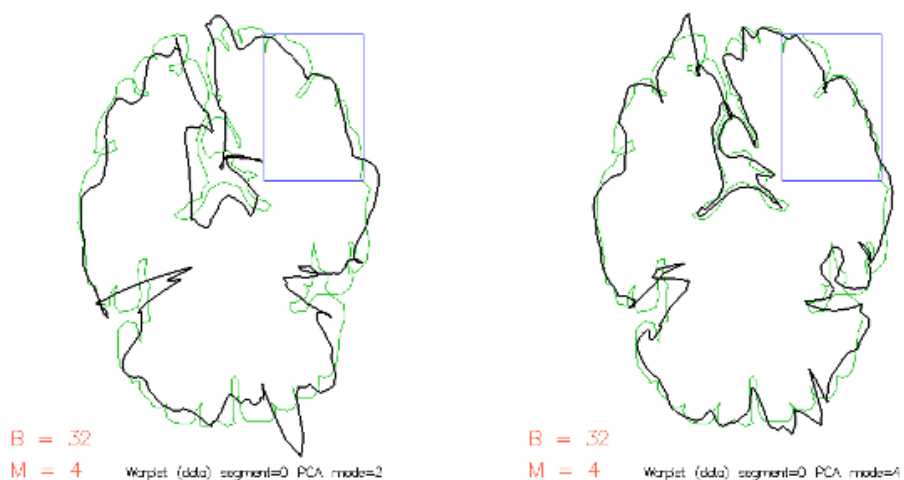

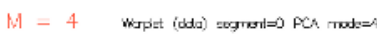
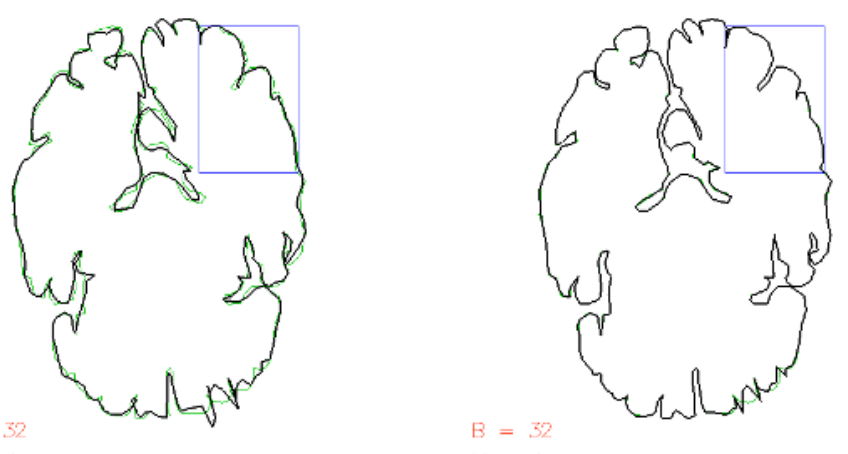

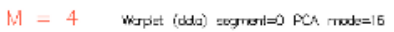

Fig. 7. Warplet reconstructions. Shown for increasing numbers of PCA modes on brain contour $D=\{0,1,2,4,8,16\}$. Point RMS errors given beside each figure

way to generate these alphabets or codebooks for sulcal-based homologies between subjects [11]. Further investigations however is needed into: extension of warplets to model surfaces by the use of spherical polar coordinates and overlapping, windowed patches of mesh points; the choice an appropriate prototypical patch, perhaps using an iterative learning approach based on residual discrpan- 
cies in shape space [7]; the variation of the contour lengths/patch sizes, possibly using a pyramid decomposition; and the constraints on the warps, e.g. to be diffeomorphic [12].

\section{References}

1. T. Cootes and C. Taylor. Active Shape Models. In Proceedings of British Machine Vision Conference, pages 265-275, 1992.

2. T. McInerney and D. Terzopoulos. Deformable models in medical image analysis: a survey. Medical Image Analysis, 1(2):91-108, 1996.

3. R. H. Davies, C. J. Twining, T. F. Cootes, J. C. Waterton, and C. J. Taylor. 3D Statistical Shape Models Using Direct Optimisation of Description Length. In Proceedings of ECCV 2002, volume 3, pages 3-20, 2002.

4. C. Davatzikos, X. Tao, and D. Shen. Hierarchical Active Shape Models, Using the Wavelet Transform. IEEE Transactions on Medical Imaging, 22(3):414-423, 2003.

5. B. C. Vermuri and A. Radisavljevic. Multiresolution Stochastic Hybrid Shape Models with Fractal Priors. ACM Transaction on Graphics, 13(2):177-207, 1994.

6. J. A. Schanabel and S. R. Arridge. Active Shape Focusing. Image and Vision Computing, 17(5-6):419-429, 1999.

7. I. Dryden. General shape and registration analysis. In O. E. Barndorff-Nielsen, W. S. Kendall, and M. N. M. van Lieshout, editors, Stochastic Geometry. Chapman and Hall, 1999.

8. I. Corouge, S. Gouttard, and G. Gerig. A Statistical Shape Model of Individual Fiber Tracts Extracted from Diffusion Tensor MRI. In Proceedings of MICCAI 2004, volume 3, pages 671-679, 2004.

9. P. M. Thompson, R. P. Woods, M. S. Mega, and A. W. Toga. Mathematical/Computational Challenges in Creating Deformable and Probabilistic Atlases of the Human Brain. Human Brain Mapping, 9:81-92, 2000.

10. L. R. Monteiro. Multivariate Regression Models and Geometric Morphometrics: The Search for Causal Factors in the Analysis of Shape. Systems Biology, 4(1):192199, 1999.

11. J.-F Mangin, D. Riviere, A. Cachia, E. Duchesnay, Y. Cointepas, D. PapadopoulosOrfanos, P. Scifo, and T. Ochiai. A framework to study the cortical foldingpatterns. Neuroimage, 23:129-138, 2004.

12. T. Cootes, C. J. Twining, and C. J. Taylor. Diffeomorphic Statistical Shape Models. In Proceedings of BMVC 2004, volume 1, pages 447-456, 2004. 